\title{
ON CONSISTENCY CHECKING OF SPATIAL RELATIONSHIPS IN CONTENT-BASED IMAGE DATABASE SYSTEMS
}

\author{
QING-LONG ZHANG*, SHI-KUO CHANG ${ }^{\dagger}$, AND STEPHEN S.-T. YAU ${ }^{\ddagger}$
}

\begin{abstract}
In this paper we investigate the consistency problem for spatial relationships in content-based image database systems. We use the mathematically simple matrix representation approach to present an efficient (i.e., polynomial-time) algorithm for consistency checking of spatial relationships in an image.

It is shown that, there exists an efficient algorithm to detect whether, given a set $\boldsymbol{S R}$ of absolute spatial relationships, the maximal set of $\boldsymbol{S} \boldsymbol{R}$ under $\mathcal{R}$ contains one pair of contradictory spatial relationships. The time required by it is at most a constant multiple of the time to compute the transitive reduction of a graph or to compute the transitive closure of a graph or to perform Boolean matrix multiplication, and thus is always bounded by time complexity $O\left(n^{3}\right)$ (and space complexity $O\left(n^{2}\right)$ ), where $n$ is the number of all involved objects. As a corollary, this detection algorithm can completely answer whether a given set of three-dimensional absolute spatial relationships is consistent.
\end{abstract}

1. Introduction. With the interest in multimedia systems over the past 10 years, content-based image retrieval has attracted the attention of researchers across several disciplines [13]. Applications that use image databases include office automation, computer-aided design, robotics, geographic data processing, remote sensing and management of earth resources, law enforcement and criminal investigation, medical pictorial archiving and communication systems, and defense. One of the most important problems in the design of image database systems is how images are stored in the image databases $[5,6,7,8]$. Various methods on image representation and retrieval can be found in the literature (see, e.g., $[4,7,8,9,10,11,12,14,15,16]$ ).

One obvious distinction between the work of Sistla et al. [16] and the work such as $[8,12]$ is that the spatial operators in $[16]$ are defined by absolute spatial relationships among objects, while the spatial operators in the other approaches are defined by relative spatial relationships among objects. Consider, for example, two significant objects $A$ and $B$ in a real picture. Then the spatial relationship " $A$ is left of $B$ " (written as " $A$ left-of $B$ ") in [8] means that the position of the centroid of $A$ is left of that of $B$ (and we say "A left-of $B$ " is relative), whereas in [16] it means that $A$

\footnotetext{
${ }^{*}$ Control and Information Laboratory, Department of Mathematics. Statistics, and Computer Science, University of Illinois at Chicago, 322 Science and Engineering Offices, 851 South Morgan Street, Chicago, Illinois 60607, USA. E-mail: zhangq@math.uic.edu

${ }^{\dagger}$ Department of Computer Science, University of Pittsburgh, Pittsburgh, PA 15260, USA. E-mail: chang@cs.pitt.edu

${ }^{\ddagger}$ Control and Information Laboratory, Department of Mathematics. Statistics, and Computer Science, University of Illinois at Chicago, 322 Science and Engineering Offices, 851 South Morgan Street, Chicago, Illinois 60607, USA. E-mail: yau@uic.edu
} 
is absolutely left of $B$ (and we say " $A$ left-of $S$ " is absolute). Note that the operator left-of has the weaker meaning in [8] than in [16] in the sense that "A left-of $B$ " is true in [8] whenever it is true in [16], and " $A$ left-of $B$ " is not necessarily true in [16] when it is true in [8]. Spatial relationships may be classified into directional and topological relationships. The $2 \mathrm{D}$ string approach developed by Chang et al. [8] is based on (relative) directional spatial relationships: left-of, right-of, above, and below. Spatial relationships used in [16] are (absolute) directional or (absolute) topological. Spatial relationships proposed in our work $[17,18,19,20,22]$ are more general, can be (absolute) directional, (relative) directional, or (absolute) topological.

In [21]. we formulated a model for Content-based Image Database Systems (CIDBS) and, for the first time, addressed the important consistency problem about content-based image indexing and retrieval. In this paper, we intend to investigate the consistency problem for spatial relationships in an image.

The rest of this paper is organized as follows. In Section 2, we briefly present the framework for Content-based Image Database Systems (CIDBS), introduced in our recent paper [21]. We demonstrate how a content-based image database system performs content-based image indexing and retrieval. In Section 3, we concentrate on investigating the consistency checking component, which is used to verify the consistency of content-based information about pictures. An efficient (i.e., polynomial-time) algorithm is given to solve the consistency problem for spatial relationships in an image. Conclusions and future research are given in Section 4.

2. Content-based Image Database Systems. In this section we briefly present the framework for Content-based Image Database Systems (CIDBS), introduced in our recent paper [21].

A Content-based Image Database System (CIDBS) will consist of at least the following seven major components: Image Capture Mechanism, Consistency Checking Mechanism, Image Indexing, Spatial Reasoning, Database, Image Matching, and Human-Computer Interface.

Figure 1 is the block diagram of a Content-based Image Database System (CIDBS). In this Figure 1, the left-side part represents an image indexing flow while the right-side part represents an image retrieval flow.

2.1. Image Indexing Flow. In this Section, we demonstrate how a Contentbased Image Database System (CIDBS) performs the image indexing work for a real picture.

For a real picture as an input, the Human-Computer Interface in a CIDBS first sends a request for capturing the picture to the Image Capture Component. The Image Capture Component will then invoke the Image Capture Mechanism to generate the content-based meta-data information about the picture. With limitations 


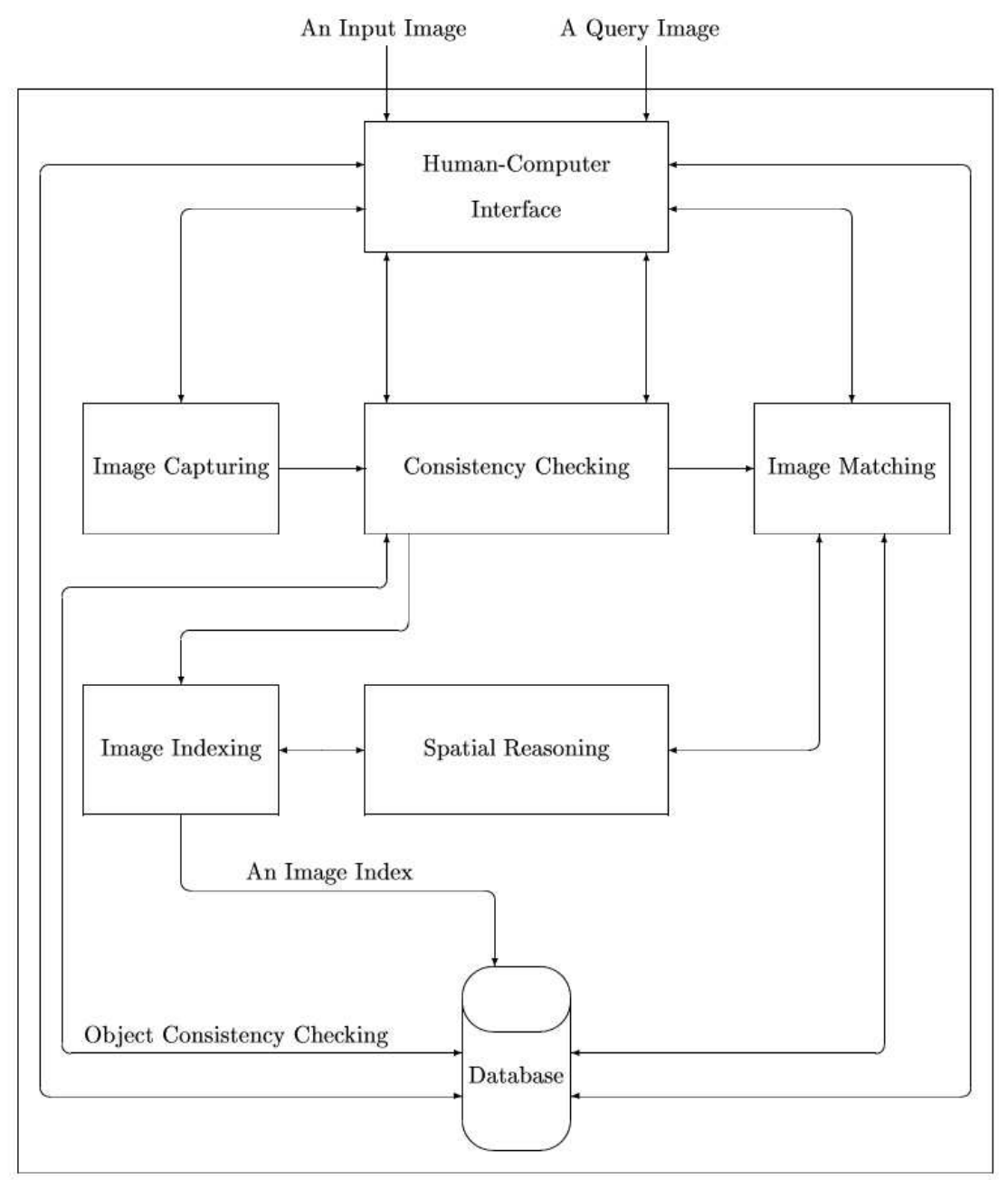

Fig. 1. Block Diagram of a content-based image database system.

of existing image-processing algorithms, this meta-data information is possibly generated semi-automatically by image-processing algorithms with human being's help or completely manually, through the Human-Computer Interface.

After the meta-data about the picture is captured, the Image Capture Component will send this meta-data to the Consistency Checking Component. The Consistency Checking Mechanism will then be invoked to verify the consistency of meta-data across the entire Database (so this step will involve the Database Component). It will perform the consistency checking among only those spatial relationships in this meta-data for the picture, while performing the consistency checking of objects in this meta-data across the entire Database.

If certain inconsistency in the meta-data is detected, the Consistency Checking 
Mechanism will temporarily stop and this inconsistency will be reported to the humanbeing for special assistance through the Human-Computer Interface. This possibly requires more accurate image-processing algorithms and/or careful manual help to recapture the picture until the inconsistency in the meta-data about that picture is solved. Certain inconsistency in the meta-data may also be detected and corrected automatically by the Consistency Checking Mechanism if the Consistency Checking Component is equipped with certain special recovery procedures. After the consistency of meta-data is verified, the Consistency Checking Component will send this meta-data to the Image Indexing Component.

After the meta-data about the picture is received, the Image Indexing Component will generate the image index for that picture based on this meta-data. The Deduction and Reduction Mechanism in the Spatial Reasoning component will also be invoked to generate the compact/minimal image index at the Image Indexing stage. Our iconic indexing approach will generate the $2 \mathrm{D}$ string representation for the image as an image index.

After an image index for the picture is produced, the Image Indexing Component will send the image index to the Database Component. Database Management System will place the image index (e.g., the 2D string representation for our iconic indexing approach) for the picture and its physical image to the database repository. An Acknowledgment of Completion message will be sent from the Database to the HumanComputer Interface to indicate the completion of image indexing for the input picture.

This finishes the image indexing flow.

2.2. Image Retrieval Flow. In this Section, we demonstrate how a Contentbased Image Database System (CIDBS) performs the image retrieval work for an image query.

An image query is inputted through the Human-Computer Interface to the Consistency Checking Component. The Consistency Checking Mechanism will be invoked to verify the consistency among spatial relationships in the content-based description of the query image. Note that it is not necessary to check the consistency among objects in the content-based description of the query image. If certain inconsistency among spatial relationships is detected, the error will be reported to the user through the Human-Computer Interface for correction of the image query. After the inconsistency among spatial relationships is resolved, the user may resubmit the modified image query through the Human-Computer Interface.

Note that, using a visual representation of an image query in the Human-Computer Interface sometimes might avoid the inconsistent problem of spatial relationships in the query, since the visual representation automatically preserves the consistency of its spatial relationships. Then it is proposed that the User Interface will have a 
mechanism to support the consistent query formulation from the visual representation of an image query.

After the consistency among spatial relationships is verified, the image query will be sent to the Image Matching Component. The query-processing mechanism will then be invoked to perform picture-matching between the query image and an image fetched from the Database, based on their content-based meta-data information. This picture-matching process may also invoke the Deduction and Reduction Mechanism in the Spatial Reasoning component to regenerate the information about redundant spatial relationships. Finally, a finite set (possibly null) of images matching the query image will be sent to the Human-Computer Interface.

This finishes the image retrieval flow.

\section{The Consistency Problem for Spatial Relationships in a Picture. In} this Section, we concentrate on investigating the consistency checking component, which is used to verify the consistency of content-based information about pictures. Specifically, we are going to present an efficient algorithm to solve the consistency problem for spatial relationships in a picture.

\subsection{The Rules for Reasoning about Absolute Spatial Relationships.}

Here first recall the semantic definitions of absolute spatial relationships, introduced in $[16]$.

It is assumed that a three-dimensional picture $p$ consists of finitely many objects and each object in $p$ corresponds to a nonempty set of points in the three-dimensional Cartesian space (the left-handed coordinate system), where each point is given by its three $x$-, $y$ - and $z$-coordinates. Given an object $X$ in a picture $p, p(X)$ denotes its corresponding nonempty set of points. A two-dimensional picture is defined similarly. Let $p$ be a picture in which objects $A$ and $B$ are contained. Now define when $p$ satisfies the following absolute spatial relationships involving basic spatial relationship operators, left-of, right-of, above, below, behind, in-front-of, inside, outside, and overlaps.

- $p$ satisfies the relationship $A$ left-of $B$, stating that $A$ is to the left of $B$ in the picture $p$, iff the $x$-coordinate of each point in $p(A)$ is less than the $x$-coordinate of each point in $p(B)$.

- $p$ satisfies the relationship $A$ above $B$, stating that $A$ is above $B$ in the picture $p$, iff the $y$-coordinate of each point in $p(A)$ is greater than the $y$-coordinate of each point in $p(B)$.

- $p$ satisfies the relationship $A$ behind $B$, stating that $A$ is behind $B$ in the picture $p$, iff the $z$-coordinate of each point in $p(A)$ is greater than the $z$ coordinate of each point in $p(B)$.

- $p$ satisfies the relationship $A$ inside $B$, stating that $A$ is inside $B$ in the picture 
$p$, iff $p(A) \subseteq p(B)$.

- $p$ satisfies the relationship $A$ outside $B$, stating that $A$ is outside $B$ in the picture $p$, iff $p(A) \cap p(B)=\emptyset$.

- $p$ satisfies the relationship $A$ overlaps $B$, stating that $A$ overlaps $B$ in the picture $p$, iff $p(A) \cap p(B) \neq \emptyset$.

The semantics of spatial relationship symbols right-of, below, and in-front-of are defined similarly. Notice that these relationship symbols right-of, below, and in-frontof are actually duals of left-of, above, and behind, respectively.

A finite set of spatial relationships $\boldsymbol{F}$ is said to be consistent if there is a picture satisfying all the relationships in $\boldsymbol{F}$. A spatial relationship $\mathrm{r}$ is said to be implied by a finite set of spatial relationships $\boldsymbol{F}$ if every picture satisfying all the relationships in $\boldsymbol{F}$ also satisfies the relationship $r$.

A deductive rule is in the following form

$$
r:: r_{1}, r_{2}, \ldots, r_{k}
$$

where $r$ and $r_{i}(1 \leq i \leq k, k \geq 0)$ are spatial relationships. The relationship $r$ and the list of relationships $r_{1}, r_{2}, \ldots, r_{k}$ are called the head and the body of the rule, respectively. A relationship $r$ is said to be deducible in one step from a set of relationships $\boldsymbol{F}$ by using a rule, if the head of the rule is $r$ and every relationship in the body of the rule is in $\boldsymbol{F}$. Let $\mathcal{R}$ be a set of rules. A relationship $r$ is said to be deducible from a set of relationships $\boldsymbol{F}$ by using the rules in $\mathcal{R}$ if $r$ is in $\boldsymbol{F}$ or there is a finite sequence of relationships $r_{1}, r_{2}, \ldots, r_{l}=r(l \geq 1)$, such that $r_{1}$ is deducible in one step from $\boldsymbol{F}$ by using a rule in $\mathcal{R}$ and for each $2 \leq i \leq l, r_{i}$ is deducible in one step from $\boldsymbol{F} \cup\left\{r_{1}, r_{2}, \ldots, r_{i-1}\right\}$ by using a rule in $\mathcal{R}$. The sequence $r_{1}, r_{2}, \ldots, r_{l}(=r)$ is called a derivation of $r$ from $\boldsymbol{F}$ by using the rules in $\mathcal{R}$ and $k$ is called the length of this derivation.

A deductive rule is called sound if every picture satisfying all the spatial relationships in the body of the rule also satisfies the spatial relationship given by the head of the rule. A set of rules $\mathcal{R}$ is called sound if every rule in $\mathcal{R}$ is sound. A set of rules $\mathcal{R}$ is said to be complete if it satisfies the following requirement for every consistent set of spatial relationships $\boldsymbol{F}$ a spatial relationship implied by $\boldsymbol{F}$ is always deducible from $\boldsymbol{F}$ by using the rules in $\mathcal{R}$.

Now let us present the system of rules $\mathcal{R}$ rules I-VIII, introduced in [16], for reasoning about absolute spatial relationships.

I. (Transitivity of left-of, above, behind, and inside) For each $x \in\{$ left-of, above, behind, inside\}, we have

$A x C:: A x B, B x C$

II. For each $x \in\{$ left-of, above, behind $\}$, we have $A \times D:: A \times B, B$ overlaps $C, C \times D$ 
III. For each $x \in\{$ left-of, above, behind, outside $\}$, we have the following two types of rules.

(a) $A x C::$ A inside $B, B x C$

(b) $A x C:: A x B, C$ inside $B$

IV. (Symmetry of overlaps and outside) For each $x \in\{$ overlaps, outside $\}$, we have $A x B:: B x A$

V. For each $x \in\{$ left-of, above, behind $\}$, we have

A outside $B:: A x B$

VI. $A$ overlaps $B:: A$ inside $B$

VII. A overlaps $B:: C$ inside $A, C$ overlaps $B$

VIII. $A$ inside $A$ ::

For two-dimensional pictures, one does not have the spatial relationship symbol behind and the rules referring to it.

Notice that, the relationship symbols right-of, below, and in-front-of are excluded in the above rules of $\mathcal{R}$, since they are duals of left-of, above, and behind, respectively. They can be handled by additional rules that simply relate them to their duals (see rules IX-XI in [16]).

Sistla et al. [16] proved that the set of rules $\mathcal{R}$ given above is sound for twodimensional and three-dimensional pictures, and $\mathcal{R}$ is complete for three-dimensional pictures. However, they presented a counterexample to show that $\mathcal{R}$ is incomplete for two-dimensional connected pictures (Note that the connectedness requirement prevents an object in a picture from having disjoint parts). Without the connectedness assumption, $\mathcal{R}$ can also be shown to be complete for two-dimensional pictures.

Unless it is otherwise stated, $\mathcal{R}$ will be used to represent the set of rules I-VIII given above.

3.2. Definitions and Basic Facts. In this Section we present some concepts, notations, definitions, and basic facts.

3.2.1. Maximal Sets of Spatial Relationships. Without loss of generality, we can assume that, for a set of spatial relationships $\boldsymbol{E}$, the maximal set of $\boldsymbol{E}$ defined below involves only those objects appearing in $\boldsymbol{E}$. Now we give the definition of the maximal set.

Definition 3.1. Given a set $\boldsymbol{E}$ of spatial relationships, a superset $\boldsymbol{F} \supseteq \boldsymbol{E}$ is called a maximal set of $\boldsymbol{t} \boldsymbol{t} \boldsymbol{E}$ under the system of rules $\mathcal{R}$ if (i) each $r \in \boldsymbol{F}$ is deducible from $\boldsymbol{E}$ using the rules in $\mathcal{R}$, and (ii) no proper superset of $\boldsymbol{F}$ satisfies condition (i).

Proposition 3.2 establishes the existence and uniqueness of the maximal set.

Proposition 3.2. Given a set $\boldsymbol{E}$ of spatial relationships, there exists exactly one maximal set $\boldsymbol{F}$ of $\boldsymbol{E}$ under $\mathcal{R}$. 
Proof. For each possible relationship $A x B$, where objects $A$ and $B$ appear in $\boldsymbol{E}$ and $x \in\{$ left-of, above, behind, inside, outside, overlaps $\}$, we put it into $\boldsymbol{F}$ if and only if it is deducible from $\boldsymbol{E}$ under $\mathcal{R}$. Then $\boldsymbol{F}$ satisfies the required properties.

Proposition 3.3 establishes the close connection of consistency between a set $\boldsymbol{E}$ of spatial relationships and the maximal set of $\boldsymbol{E}$ under $\mathcal{R}$.

Proposition 3.3. Given a set $E$ of spatial relationships, $\boldsymbol{E}$ is consistent if and only if the maximal set of $\boldsymbol{E}$ under $\mathcal{R}$ is consistent.

Proof. It is obvious that $\boldsymbol{E}$ is consistent if the maximal set of $\boldsymbol{E}$ under $\mathcal{R}$ is consistent. Conversely, if $\boldsymbol{E}$ is consistent, then the maximal set of $\boldsymbol{E}$ under $\mathcal{R}$ must be consistent, since the set of rules $\mathcal{R}$ is sound for two-dimensional and three-dimensional pictures.

3.2.2. Directed Graph and Transitive Closure. A directed graph (or digraph $G$ ) is a subset of $V \times V$, where $V$ is a finite set. The elements in $V$ and $G$ are called the vertices and arcs of the graph, respectively. Given two vertices $u$ and $v$ in $V$, a directed path in $G$ from $u$ to $v$ is a sequence of distinct $\operatorname{arcs} \alpha_{1}, \alpha_{2}, \ldots, \alpha_{k}(k \geq 1)$, such that there exists a corresponding sequence of vertices $u=v_{0}, v_{1}, v_{2}, \ldots, v_{k}=v$ satisfying $\alpha_{i+1}=\left(v_{i}, v_{i+1}\right) \in G$, for $0 \leq i \leq k-1$. A cycle is a directed path beginning and ending at the same vertex and passing through at least one other vertex. An arc in the form $(v, v)$ is called a loop. A graph is called acyclic if it contains no cycles or loops.

A graph $G$ is called transitive if, for every pair of vertices $u$ and $v$, not necessarily distinct, $(u, v) \in G$ whenever there exists a directed path in $G$ from $u$ to $v$. The transitive closure $G^{T}$ of $G$ is the least subset of $V \times V$ that contains $G$ and is transitive.

The following fact 3.4 is stated in [17, Chapter 2] [23].

FACT 3.4. It takes the same equivalent time complexity to compute the transitive reduction of a graph, or to compute the transitive closure of a graph, or to perform Boolean matrix multiplication.

Notice that we can easily compute the transitive closure of a graph $G$ using efficient standard algorithms with time complexity $O\left(n^{3}\right)$ and space complexity $O\left(n^{2}\right)$, where $n$ is the total number of vertices in $G$ (see, e.g., $[1,2,3]$ ).

Let $G$ be a directed graph. We will use $G^{T}$ to denote the transitive closure of $G$. It is assumed that a directed graph $G$ is represented by its adjacency matrix $M$, the matrix with a 1 in row $i$ and column $j$ if there is an arc from the $i$ th vertex to the $j$ th vertex and a 0 there otherwise. For simplicity, sometimes we identify a graph $G$ with its adjacency matrix $M$, and also use $M^{T}$ to denote adjacency matrix of the transitive closure $G^{T}$. For a set $\boldsymbol{E}$ of " $x$ " relationships, where $x \in\{$ left-of, above, behind, inside, outside, overlaps $\}$, we also associate it with its adjacency matrix, the matrix with a 1 in row $i$ and column $j$ if the relationship "(the $i$ th object) $x$ (the $j$ th object)" is in 
$\boldsymbol{E}$ and a 0 there otherwise, and identify $\boldsymbol{E}$ with its adjacency matrix. However, the intended meaning will be clear from the context.

Let $\boldsymbol{S R}$ be a set of spatial relationships and $n$ be the number of all objects involved in $\boldsymbol{S R}$. We assume that these $n$ objects involved in $\boldsymbol{S} \boldsymbol{R}$ are always arranged in some order from first to $n$ th. Note that, two identical objects located in different positions in a real picture are represented by different subscripts among $1,2, \ldots, n$. This is required for the description of spatial relationships and the $2 \mathrm{D}$ string representation of a picture. Certainly they will be matched to the same object during pictorial retrieval.

DEFINITION 3.5. Let $\boldsymbol{S} \boldsymbol{R}$ be a set of spatial relationships and $x$ be a relationship symbol chosen from \{left-of, above, behind, inside\}. A dependency graph derived by $x$ (and $\boldsymbol{S R}$ implicitly) is defined as a directed graph $G_{x}$, its vertex set is the set of all objects involved in $\boldsymbol{S R}$, and an arc $(A, B)$ is in $G_{x}$ if and only if $A x B$ is in $\boldsymbol{S R}$.

Note that, from Rule VIII, any relationship $A$ inside $A$ is always redundant for any involved object $A$ and thus could be deleted from $\boldsymbol{S} \boldsymbol{R}$ immediately. Further, all of them must be added into the maximal set of $\boldsymbol{S} \boldsymbol{R}$ when we generate it. Therefore, we can assume that the derived dependency graph $G_{\text {inside }}$ does not include any $\operatorname{arc}(A, A)$. Now it is obvious that four derived dependency graphs, $G_{l e f t-o f}, G_{a b o v e}, G_{b e h i n d}$, and $G_{\text {inside }}$ are acyclic for any consistent set $\boldsymbol{S} \boldsymbol{R}$ of spatial relationships.

Let $\boldsymbol{E}$ be a set of spatial relationships and $x$ be a relationship symbol. We will use $\boldsymbol{E}^{x}$ to denote the subset of all " $x$ " relationships that are in $\boldsymbol{E}$. For example, if $\boldsymbol{E}=\{A$ left-of $B, B$ left-of $C, A$ outside $C\}$, then $\boldsymbol{E}^{\text {left-of }}=\{A$ left-of $B, B$ left-of $C\}, \boldsymbol{E}^{\text {outside }}=\{A$ outside $C\}$, and $\boldsymbol{E}^{\text {inside }}=\emptyset$. Let $\boldsymbol{F}$ be a set of spatial relationships involving only overlaps or outside. We will use $\boldsymbol{F}^{s}$ to denote the set of all corresponding symmetrical relationships from $\boldsymbol{F}$. For example, if $\boldsymbol{F}_{1}=\{A$ overlaps $B, C$ overlaps $D, D$ overlaps $C\}$, then $\boldsymbol{F}_{1}^{s}=\{B$ overlaps $A, D$ overlaps $C, C$ overlaps $D\}$, and if $\boldsymbol{F}_{2}=\{A$ outside $B, C$ outside $D\}$, then $\boldsymbol{F}_{2}^{s}=\{B$ outside $A, D$ outside $C\}$.

3.3. Consistency Checking Algorithms. Now we begin to present the algorithms for consistency checking of spatial relationships.

The 2D string approach for Iconic Indexing developed by Chang et al. [8] considers only relative spatial relationships among objects, that is, it considers only relative spatial relationships involving left-of, above, and behind (for three-dimensional pictures only). Our proposed GC-2D string approach [19, 22] considers both relative and absolute spatial relationships. Note that there are no interactions among left-of, above, and behind relationships. Let us consider a set of only relative spatial relationships $\boldsymbol{E}$. We can detect the consistency of $\boldsymbol{E}$ in the following way. First, check whether $\boldsymbol{E}$ contains one self-contradictory relationship $A x A$ for some object $A$ involved in $\boldsymbol{E}$ 
and $x \in\{$ left-of, above, behind $\}$. It is obvious that $\boldsymbol{E}$ is inconsistent if $\boldsymbol{E}$ contains one self-contradictory relationship $A x A$. Now if $\boldsymbol{E}$ doesn't contain any self-contradictory relationship $A x A$, then compute the transitive closure $G_{x}^{T}$ of $G_{x}$ for each $x \in\{$ left-of, above, behind $\}$, where $G_{x}$ is the dependency graph derived by $x$ (and $\left.\boldsymbol{E}\right)$. It is clear that $\boldsymbol{E}$ is inconsistent if and only if $G_{x}$ is cyclic, if and only if $G_{x}^{T}$ contains a loop $(A, A)$ for some object $A$ involved in $\boldsymbol{E}$, if and only if $G_{x}^{T}$ contains two $\operatorname{arcs}(A, B)$ and $(B, A)$ for two different objects $A$ and $B$ involved in $\boldsymbol{E}$, where $x$ is either left-of, above, or behind. Note that the required time complexity is dominated by applying the transitive closure algorithm. Therefore, we have the following theorem.

THEOREM 3.6. There exists an efficient algorithm to detect whether a given set of relative spatial relationships $\boldsymbol{E}$ is consistent. The time required by it is at most a constant multiple of the time to compute the transitive closure of a graph, and thus is always bounded by time complexity $O\left(n^{3}\right)$ (and space complexity $O\left(n^{2}\right)$ ), where $n$ is the number of all objects involved in $\boldsymbol{E}$.

Let $\boldsymbol{E}$ be a set of spatial relationships among objects in the content-based metadata information about a picture. Note that inside, outside, and overlaps operators are not applicable for relative spatial relationships, and an absolute spatial relationship involving left-of, above, and behind is also true as a corresponding relative spatial relationship. Thus, in order to verify the consistency of $\boldsymbol{E}$, we need to do the following two consistency checkings. One is to check the consistency of the set of those absolute spatial relationships in $\boldsymbol{E}$. The rest of the paper is devoted to this. The other is to check the consistency of the union set of relative spatial relationships already in $\boldsymbol{E}$ and those corresponding relative spatial relationships which, as absolute spatial relationships, are in the maximal set of $\boldsymbol{E}$ under $\mathcal{R}$. By Theorem 3.6, this can be done efficiently as shown above.

Similar to Theorem 3.6, we clearly have the following theorem for detecting the consistency of relative and/or absolute spatial relationships involving only left-of, above, and behind.

TheOREM 3.7. There exists an efficient algorithm to detect whether a given set $\boldsymbol{E}$ of spatial relationships involving only left-of, above, and behind operators is consistent. The time required by it is at most a constant multiple of the time to compute the transitive closure of a graph, and thus is always bounded by time complexity $O\left(n^{3}\right)$ (and space complexity $O\left(n^{2}\right)$ ), where $n$ is the number of all objects involved in $\boldsymbol{E}$.

From now on, let us consider only absolute spatial relationships in the meta-data information about a picture.

Given two different objects $A$ and $B$, we say $A$ and $B$ have a pair of contradictory spatial relationships if at least one of the following six conditions holds:

1. $A$ inside $B$ and $B$ inside $A$.

2. $A x B$ and $B x A$ for some $x \in\{$ left-of above, behind $\}$. 
3. $A$ outside $B$ and $A$ overlaps $B$.

4. A overlaps $B$ and $A x B$ for some $x \in\{$ left-of above, behind $\}$.

5. $A$ inside $B$ and $A x B$ for some $x \in\{$ left-of above, behind $\}$.

6. A outside $B$ and $A$ inside $B$.

Each condition is respectively called type- $i$, where $1 \leq i \leq 6$. (Note that these are all possible cases of contradictory pairs.)

Given a set $\boldsymbol{E}$ of absolute spatial relationships, we say $\boldsymbol{E}$ contains one pair of contradictory spatial relationships if there exist two objects $A$ and $B$ having a pair of contradictory spatial relationships in $\boldsymbol{E}$. We say $\boldsymbol{E}$ contains a self-contradictory spatial relationship if there exists one object $A$ such that $\boldsymbol{E}$ contains either one of the following spatial relationships: A left-of A, A above $A, A$ behind $A$, and $A$ outside A.

It is obvious that any set $\boldsymbol{E}$ of absolute spatial relationships is inconsistent if $\boldsymbol{E}$ contains one pair of contradictory spatial relationships. It is also obvious that $\boldsymbol{E}$ is inconsistent if $\boldsymbol{E}$ contains a self-contradictory spatial relationship.

Given a set $\boldsymbol{S R}$ of absolute spatial relationships, we will follow the process of generating the maximal set of $\boldsymbol{S R}$ under $\mathcal{R}$ (see [17, Chapter 2] [23]), to detect whether the maximal set of $\boldsymbol{S} \boldsymbol{R}$ under $\mathcal{R}$ contains one pair of contradictory spatial relationships. And if the maximal set of $\boldsymbol{S R}$ tinder $\mathcal{R}$ doesn't contain any pair of contradictory spatial relationships, our proposed procedure will finally generate the maximal set of $\boldsymbol{S} \boldsymbol{R}$ under $\mathcal{R}$.

At the beginning of the process and after each step of generating certain new spatial relationships, we will check whether there exists one pair of contradictory spatial relationships so far. If the answer is YES, the maximal set of $\boldsymbol{S} \boldsymbol{R}$ under $\mathcal{R}$ definitely contains one pair of contradictory spatial relationships. If the answer is $\mathrm{NO}$, continue the process.

Before the beginning of detection algorithm, first check whether $\boldsymbol{S R}$ contains a self-contradictory spatial relationship. If $\boldsymbol{S} \boldsymbol{R}$ contains the spatial relationship $A x A$ for some object $A$ involved in $\boldsymbol{S R}$ and $x \in\{$ left-of, above, behind, outside $\}$, then $\boldsymbol{S} \boldsymbol{R}$ is inconsistent. Also note that, from Rules VIII and VI, any relationships $A$ inside $A$ and $A$ overlaps $A$ are always redundant for any involved object $A$ and thus could be deleted from $\boldsymbol{S R}$ immediately. Therefore, we can assume that $\boldsymbol{S R}$ does not contain AxA for $x \in\{$ left-of, above, behind, inside, outside, overlaps $\}$.

We divide the process of generating all deducible relationships from $\boldsymbol{S R}$ under $\mathcal{R}$ into four parts: (i) generating new inside relationships; (ii) generating new overlaps relationships; (iii) generating new relationships involving left-of, above, and behind; and (iv) generating new outside relationships. Among these four parts, the first part is the easiest and the third part is the hardest.

We begin with Part (i). 
3.3.1. Generating inside Relationships. We have only rules I and VIII to deduce inside relationships. As mentioned before, $G_{\text {inside }}$ denotes the dependency graph derived by the relationship symbol inside (and $\boldsymbol{S R}$ ) which does not contain any $\operatorname{arc}(A, A)$, where $A$ is an object. Obviously the set of all deducible inside relationships is

$G_{\text {inside }}^{T} \cup\{A$ inside $A \mid A$ is any involved object $\}$,

denoted by INSIDE.

It is clear that $G_{\text {inside }}$ is inconsistent if and only if $G_{\text {inside }}$ is cyclic, if and only if $G_{\text {inside }}^{T}$ contains a loop $(A, A)$ for some object $A$ involved in $\boldsymbol{S R}$, if and only if $G_{\text {inside }}^{T}$ contains two $\operatorname{arcs}(A, B)$ and $(B, A)$ for two different objects $A$ and $B$ involved in $\boldsymbol{S R}$. Thus, we only need to check whether $G_{\text {inside }}^{T}$ contains a loop. If $G_{\text {inside }}^{T}$ contains a loop, halt the procedure and output YES. Otherwise, continue (and we know $G_{\text {inside }}$ is acyclic).

Suppose, for example, $\boldsymbol{S} \boldsymbol{R}^{\text {inside }}=\{A$ inside $B, B$ inside $C, C$ inside $A\}$. Then $G_{\text {inside }}^{T}$ contains all nine spatial relationships $Y$ inside $Z$, where $Y$ and $Z$ can be either $A, B$, or $C$. Thus, $G_{\text {inside }}\left(=\boldsymbol{S} \boldsymbol{R}^{\text {inside }}\right)$ and $\boldsymbol{S} \boldsymbol{R}$ are inconsistent.

Later we will use the set

$\boldsymbol{I N S I D E}^{+}=G_{\text {inside }}^{T}=\boldsymbol{I N S I D E}-\{A$ inside $A \mid A$ is any involved object $\}$.

Suppose, for example, $\boldsymbol{S R}^{\text {inside }}=\{A$ inside $B, B$ inside $C\}$. Then

$$
\boldsymbol{I N S I D E}^{+}=\boldsymbol{S} \boldsymbol{R}^{\text {inside }} \cup\{A \text { inside } C\} .
$$

3.3.2. Generating overlaps Relationships. We have only three rules, IV, VI, and VII, to deduce overlaps relationships.

Let $O_{0}=\boldsymbol{S} \boldsymbol{R}^{\text {overlaps }}, O_{1}=O_{0} \cup O_{0}^{s}$, and $O_{2}$ be the set of all deducible overlaps relationships from INSIDE using Rules VI and IV. $O_{1}$ and $O_{2}$ could have nonempty intersection set. Note that $O_{1} \cup O_{2}$ is the set of all deducible overlaps relationships from $O_{0} \cup \boldsymbol{I N S I D E}$ using only Rules IV and VI.

When $C$ is set to be $A$, Rule VII will become

$$
A \text { overlaps } B:: \text { A inside } A, A \text { overlaps } B
$$

and this is trivial by Rule VIII. Similarly, when $C$ is set to be $B$, Rule VII will become

$A$ overlaps $B:: B$ inside $A, B$ overlaps $B$

and this is trivial by Rule IV, VI, and VIII. Thus, we can assume that $C$ is always not equal to $A$ or $B$ whenever we apply Rule VII.

Any new deducible relationships $A$ overlaps $B$ (i.e., not in $O_{1} \cup O_{2}$ ) should have to be obtained from $O_{1} \cup O_{2}$ and $\boldsymbol{I N S I D E ^ { + }}$ using Rule VII at least once and Rule IV. Let $O_{3}$ be the set of all overlaps relationships deducible in one step from $O_{1} \cup O_{2}$ and $\boldsymbol{I N S I D E ^ { + }}$ using Rule VII, and let $O_{4}=O_{3}^{s}$, and $O_{5}$ be the set of all overlaps relationships deducible in one step from $O_{4}$ and $\boldsymbol{I N S I D E}^{+}$using Rule VII.

Suppose, for example, $\boldsymbol{S R}=\{C$ inside $A, D$ inside $B, C$ overlaps $D\}$. Then $O_{0}=$ 
$\{C$ overlaps $D\}, O_{1}=\{C$ overlaps $D, D$ overlaps $C\}, \boldsymbol{I N S I D E ^ { + }}=\{C$ inside $A, D$ inside $B\}$ and $O_{2}=\{C$ overlaps $A, A$ overlaps $C, D$ overlaps $B, B$ overlaps $D\} \cup\{z$ overlaps $z \mid z \in\{A, B, C, D\}\}$. All new deducible relationships in $O_{3}$ are $A$ overlaps $D$ and $B$ overlaps $C$, since

$$
\begin{aligned}
& A \text { overlaps } D:: C \text { inside } A, C \text { overlaps } D \\
& B \text { overlaps } C:: D \text { inside } B, D \text { overlaps } C \text {. }
\end{aligned}
$$

Hence, $O_{4}$ contains $D$ overlaps $A$ and $C$ overlaps $B$. Now all new deducible relationships in $O_{5}$ are $A$ overlaps $B$ and $B$ overlaps $A$, since

$$
\begin{aligned}
& A \text { overlaps } B:: C \text { inside } A, C \text { overlaps } B \\
& B \text { overlaps } A:: D \text { inside } B, D \text { overlaps } A .
\end{aligned}
$$

Claim 3.8. The set of all new (i.e., not in $O_{1} \cup O_{2}$ ) deducible overlaps relationships is contained in $O_{3} \cup O_{4} \cup O_{5}$. Therefore, the set of all deducible overlaps relationships is

$$
\bigcup_{i=1}^{5} O_{i}
$$

denoted by $\boldsymbol{O V E R L A P S .}$

Proof. The reader may refer to the proof of Claim 3.1 in Appendix of [23] for the proof of Claim 3.8 .

Note that, for each object $A, A$ inside $A$ is in INSIDE, so $A$ overlaps $A$ is in $O_{2}$ by Rule VI, and thus is in $\boldsymbol{O V E R L A P S}$. Let

OVERLAPS ${ }^{+}=$OVERLAPS - $\{A$ overlaps $A \mid A$ is any involved object $\}$. We will use $\boldsymbol{O} \boldsymbol{V E \boldsymbol { E }} \boldsymbol{L} \boldsymbol{A} \boldsymbol{P} \boldsymbol{S}^{+}$later.

Note that nothing abnormal will occur at this step, since the interaction between inside and overlaps relationships is consistent.

3.3.3. Generating left-of, above, and behind Relationships. We have only the first three rules, I, II, and III, to deduce relationships involving left-of, above, and behind. To apply Rule III to deduce new relationships, we should guarantee that all deducible inside relationships be generated from $\boldsymbol{S R}$. To apply Rule II to deduce new relationships, we should also guarantee that all deducible overlaps relationships be generated from $\boldsymbol{S R}$.

We now consider generating new left-of, above, and behind spatial relationships. This generating process can be divided into three steps: (a) generating those new relationships that can be deduced by using only Rule I; (b) generating those new relationships that can be deduced by using only both Rules I and II; and (c) generating those new relationships that can be deduced by using Rules I, II, and III. Since any new relationship involving left-of, above, and behind is deducible, in the presence of 
$\boldsymbol{O V E R L A P \boldsymbol { S } ^ { + }}$ and $\boldsymbol{I N S I D E ^ { + }}$ using only Rules I, II, and III, it should be generated from $\boldsymbol{S R}$ at one of steps (a), (b), and (c).

Note that Rule II will become rule I whenever $D$ is identical to $C$, and Rule III will become trivial whenever $A$ is identical to $B$ for case (a) or $B$ is identical to $C$ for case (b). Hence, we can assume that applying Rule II requires the condition " $B$ is not identical to $C$ " and applying Rule III requires the condition " $A$ is not identical to $B$ for case (a) or $B$ is not identical to $C$ for case (b)."

The generating process goes through (a), then (b), then (c), one time for each relationship symbol $x \in\{$ left-of, above, behind $\}$.

\section{Step (a) Using only Rule I}

Recall that $G_{x}$, defined in Section 3.2.2, is the dependency graph derived by $x$ (and $\boldsymbol{S R}$ implicitly). It is obvious that $G_{x}^{T}$ is the set of all " $x$ " relationships deducible by using only Rule I.

Suppose, for example, $\boldsymbol{S R}=\{A$ above $B, B$ above $C\}$. Then $G_{\text {above }}^{T}=\boldsymbol{S R} \cup\{A$ above $C\}$.

It is clear that $G_{x}$ is inconsistent if and only if $G_{x}$ is cyclic, if and only if $G_{x}^{T}$ contains a loop $(A, A)$ for some object $A$ involved in $\boldsymbol{S R}$, if and only if $G_{x}^{T}$ contains two $\operatorname{arcs}(A, B)$ and $(B, A)$ for two different objects $A$ and $B$ involved in $\boldsymbol{S R}$. Thus, we only need to check whether $G_{x}^{T}$ contains a loop. If $G_{x}^{T}$ contains a loop, halt the procedure and output YES. Otherwise, continue (and we know $G_{x}$ is acyclic).

Then check whether $G_{x}^{T} \cup \boldsymbol{O} \boldsymbol{V E R} \boldsymbol{L} \boldsymbol{A P \boldsymbol { S } ^ { + }}$ contains one pair of type-4 contradictory spatial relationships, that is, whether there exist two different objects $A$ and $B$ such that $A x B \in G_{x}^{T}$ and $A$ overlaps $B \in \boldsymbol{O V E \boldsymbol { E R }} \boldsymbol{A} \boldsymbol{A P \boldsymbol { S } ^ { + }}$. If $G_{x}^{T} \cup \boldsymbol{O V E R L A P \boldsymbol { S } ^ { + }}$ does, halt the procedure and output YES. Otherwise, continue. Note that $\boldsymbol{O} \boldsymbol{V E R}$ $\boldsymbol{L A P \boldsymbol { S } ^ { + }}$ already contains all overlaps relationships which are obtained from $\boldsymbol{I N}$ -

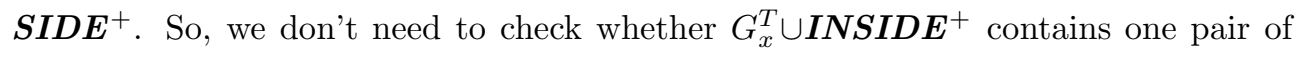
type-5 contradictory spatial relationships.

\section{Step (b) Using only Rules I and II}

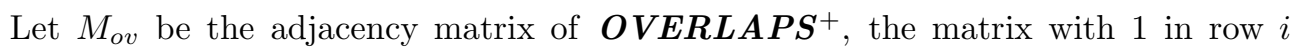
and column $j$ if the relationship "(the $i$ th object) overlaps (the $j$ th object)" is in $\boldsymbol{O V E R L A P S ^ { + }}$ and a 0 there otherwise. Then $G_{x}^{T} * M_{o v} * G_{x}^{T}$ represents the set of those " $x$ " relationships that are deducible in the presence of $\boldsymbol{O V E R L A P \boldsymbol { S } ^ { + }}$ using Rule II exactly once and Rule I zero or more times. It is easy to see that $\bigcup_{2 \leq r \leq 3}\left(G_{x}^{T} *\right.$ $\left.M_{o v}\right)^{r} * G_{x}^{T}$ represents the set of those " $x$ " relationships that are deducible in the presence of $\boldsymbol{O V E R \boldsymbol { L }} \boldsymbol{A P S ^ { + }}$ using Rule II exactly two times and Rule I zero or more times. Furthermore, $\bigcup_{r \geq 1}\left(G_{x}^{T} * M_{o v}\right)^{r} * G_{x}^{T}$ represents the set of those " $x$ " relationships that are deducible in the presence of $\boldsymbol{O V E R L A} \boldsymbol{P} \boldsymbol{S}^{+}$using Rule II at least once and Rule I zero or more times. Clearly $\bigcup_{r \geq 1}\left(G_{x}^{T} * M_{o v}\right)^{r} * G_{x}^{T}$ is the set of all new " $x$ " relationships at this step. And $\bigcup_{r \geq 0}\left(G_{x}^{T} * M_{o v}\right)^{r} * G_{x}^{T}$, denoted by $M_{x 2}$, is the set of 


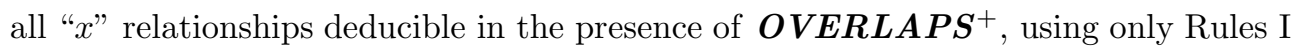
and II.

Let $M_{x}$ denote $G_{x}^{T} * M_{o v}$. It is easy to see that

$$
\begin{aligned}
M_{x 2} & =\bigcup_{r \geq 0}\left(G_{x}^{T} * M_{o v}\right)^{r} * G_{x}^{T} \\
& =G_{x}^{T}+\left(G_{x}^{T} * M_{o v}\right)^{T} * G_{x}^{T} \\
& =G_{x}^{T}+M_{x}^{T} * G_{x}^{T} .
\end{aligned}
$$

Note that $M_{x}=G_{x}^{T} * M_{o v}$ represents an "entire- $x$-partial" relation among objects, that is, $(A, C) \in G_{x}^{T} * M_{o v}$ if and only if there exists some object $B$ other than $A$ and $C$ such that $A x B$ and $B$ overlaps $C$, that means $A x(B \cap C)$, the entire object $A$ is $x$ to $B \cap C$, the part of the object $C$. This "entire- $x$-partial" relation among objects satisfies the transitive rule. Thus, $\bigcup_{r \geq 1}\left(G_{x}^{T} * M_{o v}\right)^{r}=\left(G_{x}^{T} * M_{o v}\right)^{T}=M_{x}^{T}$ is the transitive closure of $M_{x}=G_{x}^{T} * M_{o v}$. It is obvious that $M_{x 2}^{T}=M_{x 2}$.

Suppose, for example, $\boldsymbol{S} \boldsymbol{R}=\{A$ above $B, C$ overlaps $B, C$ above $D, D$ overlaps $E, E$ above $F\}$. At Step (a), $G_{\text {above }}^{T}=G_{\text {above }}=\boldsymbol{S R}^{\text {above }}$. At Step (b), OVERLAPS $\boldsymbol{S}^{+}=\{C$ overlaps $B, B$ overlaps $C, D$ overlaps $E$, $E$ overlaps $D\}$. Then

$$
\begin{aligned}
M_{\text {above }} & =G_{\text {above }} * M_{\text {ov }}=\{(A, C),(C, E)\}, \\
M_{\text {above }}^{T} & =\{(A, C),(C, E),(A, E)\}, \\
M_{\text {above }}^{T} * G_{\text {above }}^{T} & =\{A \text { above } D, C \text { above } F, \text { A above } F\} .
\end{aligned}
$$

In fact, we can have the following derivations for $A$ above $D, C$ above $F$, and $A$ above $F$ in $M_{\text {above }}^{T} * G_{\text {above }}^{T}$.

$A$ above $D:: A$ above $B, B$ overlaps $C, C$ above $D$

$C$ above $F:: C$ above $D, D$ overlaps $E, E$ above $F$

$A$ above $F:: A$ above $D, D$ overlaps $E, E$ above $F$.

Now check whether $M_{x 2}$ contains a loop. If it contains a loop, halt the procedure and output YES. Otherwise, continue (and we know $M_{x 2}$ is acyclic). Then check whether $M_{x 2} \cup M_{o v}$ contains one pair of type-4 contradictory spatial relationships, that is, whether there exist two different objects $A$ and $B$ such that $A x B \in M_{x 2}$ and $A$ overlaps $B \in M_{o v}$. If $M_{x 2} \cup M_{o v}$ does, halt the procedure and output YES. Otherwise, continue. Note that $M_{o v}$ already contains all overlaps relationships which

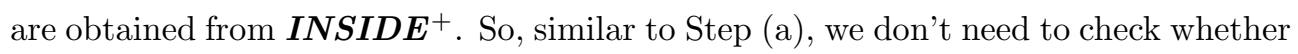
$M_{x 2} \cup \boldsymbol{I N S I D E ^ { + }}$ contains one pair of type- 5 contradictory spatial relationships.

\section{Step (c) Using Rules I, II, and III}

For the purpose of ease of disposition, here we introduce the spatial relationship symbol contains, which says that $A$ contains $B$ iff $B$ inside $A$. Let

$$
\boldsymbol{C O N T A I N S}^{+}=\left\{A \text { contains } B \mid B \text { inside } A \in \boldsymbol{I N S I D E ^ { + }}\right\} \text {, }
$$


and $M_{i n}$ and $M_{c o}$, respectively, be the adjacency matrices of the directed graphs

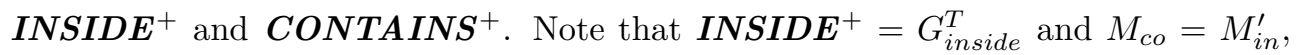
where $M_{i n}^{\prime}$ denotes the transpose matrix of $M_{i n}$.

After the spatial relationship symbol contains is introduced, Rule III(b) can be rewritten as follows:

$$
A x C:: A x B, B \text { contains } C \text {. }
$$

Now it is obvious that $M_{i n} * M_{x 2}$ and $M_{x 2} * M_{c o}$ (i.e., $M_{x 2} * M_{i n}^{\prime}$ ) represent the sets of all " $x$ " relationships deducible in one step from $M_{x 2}$ and INSIDE using Rule III(a) and Rule III(b), respectively. Furthermore, $M_{i n} * M_{x 2} * M_{c o}$ (i.e., $\left.M_{i n} * M_{x 2} * M_{i n}^{\prime}\right)$ represents the set of all " $x$ " relationships deducible in two steps

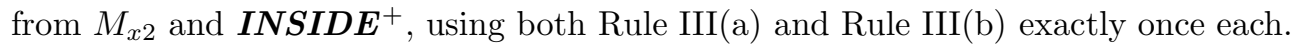
Furthermore, if $A x D \in M_{i n} M_{x 2} M_{c o}$, then

$$
\text { AxD :: } \quad A \text { inside } B, B x C, C \text { contains } D
$$

where

$$
A \text { inside } B \in \boldsymbol{I N S I D E ^ { + }}, B x C \in M_{x 2}
$$

and

$$
C \text { contains } D \in \text { CONTAINS }{ }^{+} \text {. }
$$

Note that $A x D$ can be derived by using Rule III(a) first, followed by using Rule III(b), that is,

$$
\begin{gathered}
A x C:: A \text { inside } B, B x C \\
A x D: \because A x C, C \text { contains } D
\end{gathered}
$$

and $A x D$ can also be derived by using Rule III(b) first, followed by using Rule III(a), that is,

$$
\begin{gathered}
B \times D:: B x C, C \text { contains } D \\
A \times D:: A \text { inside } B, B \times D .
\end{gathered}
$$

Suppose, for example, $\boldsymbol{S R}=\{A$ inside $B, B$ above $C, D$ inside $C, A$ above $C, A$ above $D\}$. At Step (a), $G_{\text {above }}^{T}=G_{\text {above }}=\boldsymbol{S R}^{\text {above }}$. At Step (b), OVER $\boldsymbol{R} \boldsymbol{A P \boldsymbol { S } ^ { + } =}$ $\{A$ overlaps $B, B$ overlaps $A, D$ overlaps $C, C$ overlaps $D\}, M_{\text {above }}^{T}=M_{\text {above }}=\{(A$, $D),(A, C),(B, D)\}$, and $M_{\text {above }}^{T} * M_{\text {above }}^{T}=\emptyset$. Hence, $M_{\text {above } 2}=\boldsymbol{S} \boldsymbol{R}^{\text {above }}$. This means that no above relationships are deleted from $\boldsymbol{S} \boldsymbol{R}^{\text {above }}$ at Steps (a) and (b). At Step (c), INSIDE $\boldsymbol{E}^{+}=\{A$ inside $B, D$ inside $C\}$ and $\boldsymbol{C O N T A I N \boldsymbol { S } ^ { + }}=\{B$ contains $A$, $C$ contains $D\}$.

Then $M_{\text {in }} * M_{\text {above } 2}=\{A$ above $C\}$, since

$A$ above $C:: A$ inside $B, B$ above $C$.

$M_{\text {above } 2} * M_{\text {co }}=\{B$ above $D, A$ above $D\}$, since

$B$ above $D:: B$ above $C, C$ contains $D$,

$A$ above $D:: A$ above $C, C$ contains $D$.

And $M_{\text {in }} * M_{\text {above } 2} * M_{c o}=\{A$ above $D\}$, since 
$A$ above $D:: A$ inside $B, B$ above $C, C$ contains $D$.

ClaIm 3.9. $M_{x 2} \cup M_{i n} M_{x 2} \cup M_{x 2} M_{i n}^{\prime} \cup M_{i n} M_{x 2} M_{i n}^{\prime}$, denoted by $\boldsymbol{M A X}(x)$, is the set of all " $x$ " relationships that are deducible, in the presence of $\boldsymbol{O V E \boldsymbol { E L } \boldsymbol { A P S }} \boldsymbol{S}^{+}$ and INSIDE $\boldsymbol{E}^{+}$, by using Rules I, II, and III.

Proof. The reader may refer to the proof of Claim 3.2 in Appendix of [23] for the proof of Claim 3.9.

Therefore, $M_{i n} M_{x 2} \cup M_{x 2} M_{i n}^{\prime} \cup M_{i n} M_{x 2} M_{i n}^{\prime}$ is the set of all new " $x$ " relationships at Step (c). And it is obvious that $\boldsymbol{M A} \boldsymbol{X}(x)^{T}=\boldsymbol{M} \boldsymbol{A} \boldsymbol{X}(x)$.

Claim 3.10. $\boldsymbol{M A} \boldsymbol{X}(x)$ does not contain a loop, i.e., $\boldsymbol{M A X}(x)$ is acyclic.

Proof. Assume that $\boldsymbol{M A X}(x)$ contains a loop $(A, A)$. Note that $M_{x 2}$ doesn't contain a loop from Step (b). So, either one of $M_{i n} M_{x 2}, M_{x 2} M_{i n}^{\prime}$ or $M_{i n} M_{x 2} M_{i n}^{\prime}$ contains a loop $(A, A)$. If $M_{i n} M_{x 2}$ contains a loop $(A, A$, then

$A x A::$ A inside $B, B \times A$

where $A$ inside $B \in \boldsymbol{I N S I D E ^ { + }}$ and $B x A \in M_{x 2}$.

Now $B$ overlaps $A \in \boldsymbol{O} \boldsymbol{V E R L A P \boldsymbol { S } ^ { + }}$. Thus, $M_{x 2} \cup M_{o v}$ contains one pair of type- 4 contradictory spatial relationships, $B$ overlaps $A$ and $B x A$. This is impossible because it is already checked in Step (b).

If $M_{x 2} M_{i n}^{\prime}$ contains a loop $(A, A)$, then

\section{$A x A::$ A $x$ B,A inside $B$}



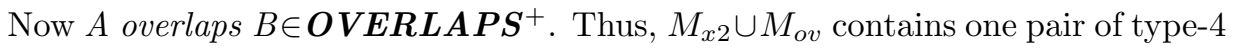
contradictory spatial relationships, $A$ overlaps $B$ and $A x B$. This is impossible because it is already checked in Step (b).

If $M_{i n} M_{x 2} M_{i n}^{\prime}$ contains a loop $(A, A)$,then

$A x A::$ A inside $B, B x C, A$ inside $C$

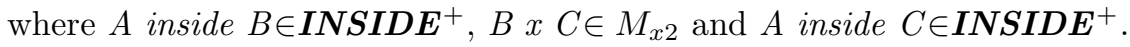

Now $A$ overlaps $C$ can be deduced from $A$ inside $C$ using Rule VI and $B$ overlaps $C$ can be deduced from $A$ inside $B$ and $A$ overlaps $C$ using Rule VII, so $B$ overlaps $C \in \boldsymbol{O V E R L A P S ^ { + }}$. Thus, $M_{x 2} \cup M_{o v}$ contains one pair of type- 4 contradictory spatial relationships, $B$ overlaps $C$ and $B x C$. This is also impossible because it is already checked in Step (b).

Thus, the assumption that $\boldsymbol{M A} \boldsymbol{X}(x)$ contains a loop $(A, A)$ is incorrect. Therefore, $\boldsymbol{M A} \boldsymbol{X}(x)$ doesn't contain a loop $(A, A)$ and $\boldsymbol{M} \boldsymbol{A} \boldsymbol{X}(x)$ is acyclic.

Now check whether $\boldsymbol{M A} \boldsymbol{X}(x) \cup M_{o v}$ contains one pair of type-4 contradictory spatial relationships, that is, whether there exist two different objects $A$ and $B$ such that $A x B \in \boldsymbol{M A X}(x)$ and $A$ overlaps $B \in M_{o v}$. If $\boldsymbol{M A} \boldsymbol{X}(x) \cup M_{o v}$ does, halt the procedure and output YES. Otherwise, continue. Note that $M_{o v}$ already contains all

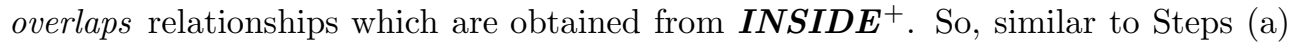




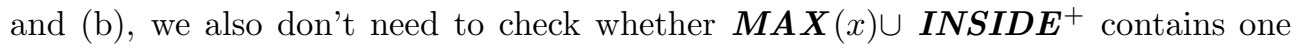
pair of type- 5 contradictory spatial relationships.

Consider, for example, $\boldsymbol{S R}=\{A$ inside $B, B$ above $C, A$ overlaps $C\}$. At Step (a), $G_{\text {above }}^{T}=G_{\text {above }}=\boldsymbol{S} \boldsymbol{R}^{\text {above }}=\{B$ above $C\}$. At Step (b), OVERLAPS $\boldsymbol{S}^{+}=\{A$ overlaps $B, B$ overlaps $A, A$ overlaps $C, C$ overlaps $A\}, M_{\text {above }}^{T}=M_{\text {above }}=\{(B, A)\}$, and $M_{\text {above }}^{T} * G_{\text {above }}^{T}=\emptyset$. Hence, $M_{\text {above } 2}=\boldsymbol{S} \boldsymbol{R}^{\text {above }}=\{B$ above $C\}$. At Step (c), IN-

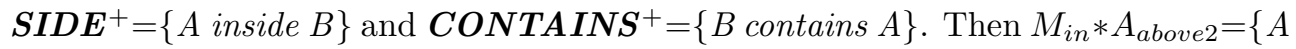
above $C$ \}, since

$A$ above $C::$ A inside $B, B$ above $C$,

$M_{\text {above } 2} * M_{c o}=\emptyset$, and $M_{i n} * M_{\text {above } 2} * M_{c o}=\emptyset$. Thus $\boldsymbol{M A} \boldsymbol{X}(x)=\{B$ above $C$, $A$ above $C$ \}. Now $\boldsymbol{M A X}(x) \cup M_{o v}$ contains one pair of type- 4 contradictory spatial relationships, $A$ above $C$ and $A$ overlaps $C$. Therefore, $\boldsymbol{S R}$ is inconsistent.

3.3.4. Generating outside Relationships. We have only three rules, III(a) (Rule III(b) is redundant for the outside relationship), IV, and V, that can be used to deduce outside relationships. Because deducing outside relationships by using Rules III(a), IV, and V is similar to deducing overlaps relationships by using Rules VII, IV, and VI. Hence, we will generate all the outside relationships from $\boldsymbol{S} \boldsymbol{R}$ similar to generating all the overlaps relationships in Section 3.3.2. We already have INSIDE from Section 3.3.1, and $\boldsymbol{M A X}(x)$ for each $x \in\{$ left-of, above, behind $\}$ from Section 3.3.3.

Let $U_{0}=\boldsymbol{S} \boldsymbol{R}^{\text {outside }}, U_{1}=U_{0} \cup U_{0}^{s}$, and $U_{2}$ be the set of all deducible outside relationships from $\boldsymbol{M} \boldsymbol{A} \boldsymbol{X}$ (left-of) $\cup \boldsymbol{M} \boldsymbol{A} \boldsymbol{X}$ (above) $\cup \boldsymbol{M} \boldsymbol{A} \boldsymbol{X}$ (behind) by using Rules $\mathrm{V}$ and IV. Then $U_{1} \cup U_{2}$ is the set of all deducible outside relationships from $U_{0}$ and $\boldsymbol{M A} \boldsymbol{X}(x)$, where $x \in\{$ left-of, above, behind $\}$, using only Rules IV and V.

Now check whether $U_{1} \cup U_{2} \cup M_{o v}$ contains one pair of type-3 contradictory spatial relationships, that is, whether there exist two different objects $A$ and $B$ such that $A$ outside $B \in U_{1} \cup U_{2}$ and $A$ overlaps $B \in M_{o v}$. If $U_{1} \cup U_{2} \cup M_{o v}$ does, halt the procedure and output YES. Otherwise, continue. Note that $M_{o v}$ already contains all overlaps

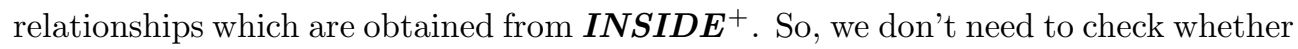
$U_{1} \cup U_{2} \cup \boldsymbol{I N S I D \boldsymbol { E } ^ { + }}$ contains one pair of type- 6 contradictory spatial relationships.

In fact, $U_{2} \cup M_{o v}$ should not contain any pair of type-3 contradictory spatial relationships. Since, so far $=\boldsymbol{M} \boldsymbol{A} \boldsymbol{X}$ (left-of) $\cup \boldsymbol{M A X}$ (above) $\cup \boldsymbol{M A X}$ (behind) $\cup M_{o v}$ does not contain any pair of type- 4 contradictory spatial relationships, this is already checked in Section 3.3.3. Thus, actually we only need to check whether $U_{1} \cup M_{o v}$ contains one pair of type- 3 contradictory spatial relationships. Since, when $B$ is identical to $A$, Rule III(a) for " $x$ " chosen as "outside" will become

$$
A \text { outside } C:: \text { A inside } A, A \text { outside } C
$$

and this is trivial by Rule VIII. Thus, we can assume that $B$ is always not identical 
to $A$ whenever we apply Rule III(a) for outside relationships.

Any new deducible relationship $A$ outside $C$ (i.e., not in $U_{1} \cup U_{2}$ ) should have to be derived from $U_{1} \cup U_{2}$ and $\boldsymbol{I N S I D \boldsymbol { E } ^ { + }}$ using Rule III(a) at least once and Rule IV. Let $U_{3}$ be the set of all outside relationships deducible in one step from $U_{1} \cup U_{2}$ and $\boldsymbol{I N S I D E ^ { + }}$ using Rule III(a), and let $U_{4}=U_{3}^{s}$ and $U_{5}$ be the set of all outside relationships deducible in one step from $U_{4}$ and $\boldsymbol{I N S I D \boldsymbol { E } ^ { + }}$ using Rule III(a). Then, we have the following claim.

Claim 3.11. The set of all new (i.e., not in $U_{1} \cup U_{2}$ ) deducible outside relationships is contained in $U_{3} \cup U_{4} \cup U_{5}$. Therefore, the set of all deducible outside relationships is

$$
\cup_{i=1}^{5} U_{i}
$$

denoted by OUTSIDE.

Proof. Similar to the proof of Claim 3.8 in Section 3.3.2, which is placed in Appendix of [23].

Let $U_{12}$ be the adjacency matrix of $U_{1} \cup U_{2}$. Then it is easy to see that $U_{3}, U_{4}$, and $U_{5}$, respectively, have the adjacency matrices $M_{i n} * U_{12},\left(M_{i n} * U_{12}\right)^{\prime}$ (i.e., $U_{12} * M_{i n}^{\prime}$. Note that $\left.U_{12}^{\prime}=U_{12}\right)$, and $M_{i n} * U_{12} * M_{i n}^{\prime}$.

Note that the proof of the following Claim 3.12 is similar to the proof of Claim 3.10 in Section 3.3.3.

Claim 3.12. OUTSIDE does not contain any self-contradictory spatial relationships A outside A, where $A$ is an object involved in $\boldsymbol{S R}$.

Proof. Note that $U_{0}=\boldsymbol{S} \boldsymbol{R}^{\text {outside }}$ doesn't contain any $A$ outside $A$, since this is already checked at the beginning of our detection procedure. So $U_{1}=U_{0} \cup U_{0}^{s}$ also doesn't contain any A outside A.

By Claim 3.10 in Section 3.3.3, $\boldsymbol{M A} \boldsymbol{X}(x)$ doesn't contain any loop $(A, A)$ for each $x \in\{$ left-of, above, behind $\}$. So $U_{2}$ doesn't contain any $A$ outside $A$.

Hence, $U_{12}=U_{1} \cup U_{2}$ doesn't contain any $A$ outside $A$.

Now assume that $\boldsymbol{O U T S I D E}$ contains a self-contradictory spatial relationship $A$ outside $A$. Then, either one of $U_{3}=M_{i n} U_{12}, U_{4}=U_{12} M_{i n}^{\prime}$, or $U_{5}=M_{i n} U_{12} M_{i n}^{\prime}$ contains a self-contradictory spatial relationship $A$ outside $A$.

If $M_{i n} U_{12}$ contains a relationship $A$ outside $A$, then $A$ outside $A::$ A inside $B, B$ outside $A$

where $A$ inside $B \in \boldsymbol{I N S I D \boldsymbol { E } ^ { + }}$ and $B$ outside $A \in U_{12}$.

Now $B$ overlaps $A \in \boldsymbol{O V E R L A P \boldsymbol { S } ^ { + }}$. Thus, $U_{12} \cup M_{o v}$ contains one pair of type-3 contradictory spatial relationships, $B$ outside $A$ and $B$ overlaps $A$. This is impossible because it is already checked earlier in this Section.

If $U_{12} M_{\text {in }}^{\prime}$ contains a relationship $A$ outside $A$, then

$$
A \text { outside } A:: A \text { outside } B: A \text { inside } B
$$


where $A$ outside $B \in U_{12}$ and $A$ inside $B \in \boldsymbol{I N S I D E} \boldsymbol{E}^{+}$.

Now $A$ overlaps $B \in \boldsymbol{O} \boldsymbol{V E R L A P \boldsymbol { S } ^ { + }}$. Thus, $U_{12} \cup M_{o v}$ contains one pair of type-3 contradictory spatial relationships, $A$ overlaps $B$ and $A$ outside $B$. This is impossible because it is already checked earlier in this Section.

If $M_{i n} U_{12} M_{i n}^{\prime}$ contains a relationship $A$ outside $A$, then

$A$ outside $A:: \quad A$ inside $B, B$ outside $C, A$ inside $C$

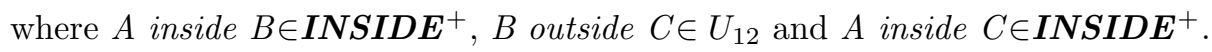

Now $A$ overlaps $C$ can be deduced from $A$ inside $C$ using Rule VI and $B$ overlaps $C$ can be deduced from $A$ inside $B$ and $A$ overlaps $C$ using Rule VII, so $B$ overlaps $C \in \boldsymbol{O V E R L A P \boldsymbol { S } ^ { + }}$. Thus, $U_{12} \cup M_{o v}$ contains one pair of type-3 contradictory spatial relationships, $B$ overlaps $C$ and $B$ outside $C$. This is also impossible because it is already checked earlier in this Section.

Thus, the assumption that $\boldsymbol{O} \boldsymbol{U T S I D E}$ contains a self-contradictory spatial relationship $A$ outside $A$ is incorrect. Therefore, $\boldsymbol{O U T S I D E}$ doesn't contain any selfcontradictory spatial relationship $A$ outside $A$.

Now check whether $\boldsymbol{O U T S I D E} \cup M_{o v}$ contains one pair of type-3 contradictory spatial relationships, that is, whether there exist two different objects $A$ and $B$ such

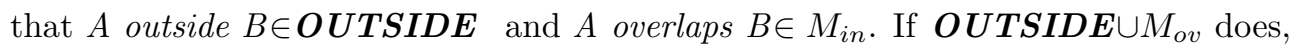
halt the procedure and output YES. Otherwise, halt the procedure and output NO. Note that $M_{o v}$ already contains all overlaps relationships which are obtained from $\boldsymbol{I N S I D E}{ }^{+}$. So, we don't need to check whether $\boldsymbol{O U T S I D E} \cup \boldsymbol{I N S I D E ^ { + }}$ contains one pair of type- 6 contradictory spatial relationships.

This completes the consistency detection procedure.

\subsubsection{Algorithm for Consistency Checking of Absolute Spatial rela-} tionships. Let $\boldsymbol{S R}$ be a set of absolute spatial relationships. It is easy to see that if the maximal set of $\boldsymbol{S R}$ under $\mathcal{R}$ doesn't contain any pair of contradictory spatial relationships, then

$\cup\{\boldsymbol{M A X}(x) \mid x \in\{$ left-of, above, behind $\}\} \cup \boldsymbol{O V E R L A P S} \cup \boldsymbol{O U T T S I D E} \cup$ INSIDE is the set of all spatial relationships deducible from $\boldsymbol{S R}$ using rules in $\mathcal{R}$, that is, the maximal set of $\boldsymbol{S R}$ under $\mathcal{R}$.

The detection algorithm, for checking whether the maximal set of $\boldsymbol{S R}$ under $\mathcal{R}$ contains one pair of contradictory spatial relationships, is summarized as follows. In this algorithm, addition ' + ' and multiplication ' $*$ ' denote Boolean matrix addition and multiplication, respectively; subtraction '-' denotes Boolean matrix subtraction corresponding to the difference operation of two sets of " $x$ " relationships, where $x \in\{$ left-of, above, behind, inside, outside, overlaps $\}$, more precisely, let $X=\left(x_{i j}\right)_{n \times n}$ and $Y=\left(y_{i j}\right)_{n \times n}$ be two $n \times n$ Boolean matrices, then $X-Y$ is an $n \times n$ Boolean matrix 
$Z=\left(z_{i j}\right)_{n \times n}$ satisfying the condition that, for $1 \leq i, j \leq n, z_{i j}=x_{i j}-y_{i j}$, where the subtraction ' - ' on two Boolean values is defined in this way: $0-0=0,0-1=0$, $1-0=1$, and $1-1=0$. Also note that, addition ' + ' and multiplication ' $*$ ' on two Boolean values are denned in the following way: $0+0=0,0+1=1,1+0=1$, and $1+1=1 ; 0 * 0=0,0 * 1=0,1 * 0=0$, and $1 * 1=1$. The following algorithm assumes that we already have efficient standard algorithm for computing the transitive closure $G^{T}$ of a given directed graph $G$. The algorithm for computing $G^{T}$ of $G$ is represented by $\operatorname{TranC}\left(G, G^{T}\right)$, where $G$ is a directed graph as input and $G^{T}$ is a directed graph as output of TranC. For each $x \in\{$ left-of, above, behind, inside, outside, overlaps $\}$, all sets of " $x$ " relationships are identified with their associated adjacency matrices. Let $I$ be an $n \times n$ identity matrix, where $n$ is the number of all objects involved in $\boldsymbol{S R}$. Then $I$ can denote either the set $\{A$ inside $A \mid A$ is any involved object $\}$ if the intended relationship is inside or the set $\{A$ overlaps $A \mid A$ is any involved object $\}$ if the intended relationship is overlaps.

Algorithm. Detect whether the maximal set of a given set of absolute spatial relationships contains one pair of contradictory spatial relationships.

Input: a given set $\boldsymbol{S R}$ of absolute spatial relationships.

Output: NO if the maximal set of $\boldsymbol{S} \boldsymbol{R}$ doesn't contain any pair of contradictory spatial relationships and the maximal set of $\boldsymbol{S} \boldsymbol{R}$ is also produced; YES, otherwise.

${ }^{*}$ Assume $\boldsymbol{S R}$ doesn't contain any AxA for $x \in\{$ left-of, above, behind, inside, outside, overlaps $\}^{*} /$

Step (0). Check whether $\boldsymbol{S R}$ contains one pair of contradictory spatial relationships. If YES, halt. Otherwise, continue.

Step (1). Generate inside relationships

$/ * G_{\text {inside }}$ denotes the dependency graph derived by inside and $\boldsymbol{S R} * /$

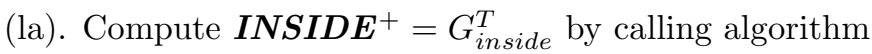
$\operatorname{TranC}\left(G_{\text {inside }}, I_{N S I D E^{+}}\right)$.

Check whether INSIDE ${ }^{+}$contains a loop.

If YES, halt. Otherwise, continue.

(lb). INSIDE $=I N S I D E^{+}+I$

Step (2). Generate overlaps relationships

$/ * O_{0}=\boldsymbol{S} \boldsymbol{R}^{\text {overlaps }}$ denotes the subset of all overlaps relationships in $\boldsymbol{S R}$ */

(2a). $O_{1}=O_{0}+O_{0}^{\prime}, O_{2}=I N S I D E+I N S I D E^{\prime}$, and set $M_{12}=O_{1}+O_{2}$;

/* $M_{\text {in }}$ is the adjacency matrix of $\mathbf{I N S I D \boldsymbol { E } ^ { + * }}$ /

(2b). $O_{3}=M_{i n}^{\prime} * M_{12}, O_{4}=O_{3}^{\prime}$ and $O_{5}=O_{3} * M_{i n}$;

(2c). OVERLAPS $=M_{12}+O_{3}+O_{4}+O_{5}$, and set OVERLAPS ${ }^{+}=$OVERLAPS- $I$. 
Step (3). Generate left-of, above, and behind relationships

$/ * G_{x}$ denotes the dependency graph derived by $x$ and $\boldsymbol{S R} * /$

For each $x \in\{$ left-of, above, behind $\}$, go through (3a)-(3c):

(3a). Compute $G_{x}^{T}$ by calling algorithm $\operatorname{TranC}\left(G_{x}, G_{x}^{T}\right)$.

Check whether $G_{x}^{T}$ contains a loop. If YES, halt. Otherwise, continue.

$/ M_{o v}$ is the adjacency matrix of $\boldsymbol{O V E R L A P \boldsymbol { S } ^ { + * } /}$

Check whether $G_{x}^{T} \cup M_{o v}$ contains one pair of type-4

contradictory spatial relationships.

If YES, halt. Otherwise, continue.

(3b). $M_{x}=G_{x}^{T} * M_{o v}$, and compute $M_{x}^{T}$ by calling algorithm

$\operatorname{TranC}\left(M_{x}, M_{x}^{T}\right)$,

then set $M_{x 2}=G_{x}^{T}+M_{x}^{T} * G_{x}^{T}$.

Check whether $M_{x 2}$ contains a loop. If YES, halt. Otherwise, continue.

Check whether $M_{x 2} \cup M_{o v}$ contains one pair of type-4

contradictory spatial relationships.

If YES, halt. Otherwise, continue.

(3c). $\boldsymbol{M A X}(x)=M_{x 2}+M_{i n} * M_{x 2}+M_{x 2} * M_{i n}^{\prime}+M_{i n} * M_{x 2} * M_{i n}^{\prime}$.

Check whether $\boldsymbol{M A} \boldsymbol{X}(x) \cup M_{o v}$ contains one pair of type-4

contradictory relationships.

If YES, halt. Otherwise, continue.

Step (4). Generate outside relationships

/* $U_{0}=\boldsymbol{S} \boldsymbol{R}^{\text {outside }}$ denotes the subset of all outside relationships in $\boldsymbol{S R} *$ /

(4a). $U_{1}=U_{0}+U_{0}^{\prime}, U_{2}=\boldsymbol{M A} \boldsymbol{X}($ left-of $)+\boldsymbol{M} \boldsymbol{A} \boldsymbol{X}($ above $)+\boldsymbol{M A} \boldsymbol{X}$ (behind)

and reset $U_{2}=U_{2}+U_{2}^{\prime}$, then $U_{12}=U_{1}+U_{2}$.

Check whether $U_{12} \cup M_{o v}$ contains one pair of type-3

contradictory spatial relationships.

If YES, halt. Otherwise, continue.

(4b). $U_{3}=M_{i n} * U_{12}, U_{4}=U_{3}^{\prime}$ and $U_{5}=M_{i n} * U_{4}$;

(4c). $\boldsymbol{O U T S I D E}=U_{12}+U_{3}+U_{4}+U_{5}$.

Check whether $\boldsymbol{O U} \boldsymbol{T S I D E} \cup U_{o v}$ contains one pair of type-3

contradictory relationships.

If YES, halt and output YES. Otherwise, halt and output NO.

/* End of the detection algorithm */

Note that if the above detection algorithm outputs YES, we are certain that $\boldsymbol{S R}$ is inconsistent. But it does not exactly tell us the questionable relationship(s) in $\boldsymbol{S} \boldsymbol{R}$ causing the inconsistency. Consider, for example, $\boldsymbol{S} \boldsymbol{R}=\{A$ above $B, B$ above $C, C$ above $A$ \}. Then $\boldsymbol{S R}$ is inconsistent. Deleting either one of the three relationships in $\boldsymbol{S R}$ will make the left two relationships in $\boldsymbol{S R}$ consistent. Thus, the user may be 
required to help resolve the inconsistency of $\boldsymbol{S R}$ when the inconsistency of $\boldsymbol{S R}$ is detected and reported to the Human-Computer Interface.

It is easy to see that, for the above algorithm, every computation at each step, excluding computing the transitive closure of a directed graph and performing Boolean matrix multiplication, can be done by time complexity $O\left(n^{2}\right)$ and space complexity $O\left(n^{2}\right)$. Notice that computing the transitive closure of a directed graph or performing Boolean matrix multiplication each has to take at least time $O\left(n^{2}\right)$. Hence, by Fact 3.4, the above algorithm will require time that is at most a constant multiple of the time to compute the transitive reduction of a graph or to compute the transitive closure of a graph or to perform Boolean matrix multiplication. Note that we can easily compute $G^{T}$ of a graph $G$, using efficient standard algorithms with time complexity $O\left(n^{3}\right)$ and space complexity $O\left(n^{2}\right)$ (see, e.g., [1, 2, 3]), and perform Boolean matrix multiplication using usual matrix multiplication with time complexity $O\left(n^{3}\right)$ and space complexity $O\left(n^{2}\right)$. Therefore, the time complexity and space complexity of the above algorithm are bounded by $O\left(n^{3}\right)$ and $O\left(n^{2}\right)$, respectively.

Now we have the following theorem.

THEOREM 3.13. There exists an efficient algorithm to detect whether, given a set $\boldsymbol{S R}$ of absolute spatial relationships, the maximal set of $\boldsymbol{S} \boldsymbol{R}$ under $\mathcal{R}$ contains one pair of contradictory spatial relationships. The time required by it is at most a constant multiple of the time to compute the transitive reduction of a graph or to compute the transitive closure of a graph or to perform Boolean matrix multiplication, and thus is always bounded by time complexity $O\left(n^{3}\right)$ (and space complexity $O\left(n^{2}\right)$ ), where $n$ is the number of all involved objects.

Given a set $\boldsymbol{S} \boldsymbol{R}$ of three-dimensional absolute spatial relationships, we can use the above algorithm to find the maximal set of $\boldsymbol{S R}$ under the system of rules $\mathcal{R}$ if the maximal set of $\boldsymbol{S} \boldsymbol{R}$ doesn't contain any pair of contradictory spatial relationships. Since $\mathcal{R}$. is complete for three-dimensional pictures, the maximal set of $\boldsymbol{S} \boldsymbol{R}$ under $\mathcal{R}$. coincides with the maximal set implied by $\boldsymbol{S R}$ under $\mathcal{R}$. Thus, it is easy to see from the entire detection procedure that $\boldsymbol{S R}$ is consistent if the above algorithm outputs NO. Therefore, we have the following corollary.

COROLlary 3.14. The above detection algorithm can completely answer whether a given set of three-dimensional absolute spatial relationships is consistent.

For two-dimensional pictures, we will not have the relationship symbol behind and the rules referring to it in $\mathcal{R}$. Similarly, we can use the above algorithm (discarding those computations involving behind relationships) to detect whether, given a set $\boldsymbol{S R}$ of two-dimensional absolute spatial relationships, the maximal set of $\boldsymbol{S} \boldsymbol{R}$ under $\mathcal{R}$. contains one pair of contradictory spatial relationships. However, since $\mathcal{R}$. is incomplete for two-dimensional connected pictures, the maximal set of $\boldsymbol{S} \boldsymbol{R}$ under $\mathcal{R}$ may not coincide with the maximal set implied by $\boldsymbol{S} \boldsymbol{R}$ under $\mathcal{R}$. More precisely, the 
maximal set of $\boldsymbol{S R}$ under $\mathcal{R}$ may be contained properly in the maximal set implied by $\boldsymbol{S} \boldsymbol{R}$ under $\mathcal{R}$. Hence, even if the above detection algorithm outputs NO, it is still possible that there exist (s) certain spatial relationship (s), implied by $\boldsymbol{S} \boldsymbol{R}$ under $\mathcal{R}$. but not deducible from $\boldsymbol{S R}$ under $\mathcal{R}$, which might cause inconsistency of $\boldsymbol{S R}$. Thus, we have the following question: Does the maximal set of an inconsistent set of planar absolute spatial relationships under $\mathcal{R}$ always contain one pair of contradictory spatial relationships or a self-contradictory spatial relationship? If the answer to this question is YES, then we can always use the above algorithm to detect the inconsistency of spatial relationships in the meta-data about a picture. Otherwise, if the answer to this question is NO, our proposed algorithm might fail to detect inconsistency of the description of absolute spatial relationships (involving inside, outside, and overlaps, by Theorem 3.7) for certain planar pictures, while at least checking their maximal sets under $\mathcal{R}$ don't contain any pair of contradictory spatial relationships.

The detailed algorithm given above can be directly programmed into executable computer codes.

4. Conclusions and Future Research. In this paper we have investigated the consistency problem for spatial relationships in Content-based Image Database Systems (CIDBS). We have used the same approach of mathematically simple matrix representation as in [23] to present an efficient (i.e., polynomial-time) algorithm to solve the consistency problem for spatial relationships in a picture. Our proposed algorithm might fail to detect inconsistency of the description of absolute spatial relationships (involving inside, outside, and overlaps) for certain planar pictures, which will require further investigation. It is straightforward to implement the detailed algorithm given in Section 3 using programming languages such as $\mathrm{C} / \mathrm{C}++$. Future research is required to further investigate the CIDBS model for facilitating fast image indexing and retrieval.

While the data consistency problem has been well addressed in traditional database systems, the consistency problem about content-based multimedia indexing and retrieval needs to be investigated. The consistency problem will also arise when multimedia data sources are merged. Our proposed approach for the image database case is an attempt to begin addressing this important issue.

\section{REFERENCES}

[1] A. V. Aho, J. E. Hopcroft, And J. D. Ullman, The Design and Analysis of Computer Algorithms, Addison-Wesley, Reading, Massachusetts, 1974.

[2] A. V. Aho, J. E. Hopcroft and J. D. Ullman, Data Structures and Algorithms, AddisonWesley, Reading, Massachusetts, 1983.

[3] S. BAAse, Computer Algorithms: Introduction to Design and Analysis, second edition, Addison-Wesley, Reading, Massachusetts, 1988. 
[4] C. C. Chang and S. Y. Lee, Retrieval of Similar Pictures on Pictorial Databases, Pattern Recognition, 24:7(1991), pp. 675-680.

[5] S.-K. Chang, Pictorial Information Systems, Prentice-Hall, Englewood Cliffs, N. J., 1989.

[6] S.-K. Chang And A. Hsu, Image Information Systems: Where do we go from here?, IEEE Transactions on Knowledge and Data Engineering, 4:5(1992), pp. 431-442.

[7] S.-K. Chang and E. Jungert, Symbolic Projection for Image Information Retrieval and Spatial Reasoning, Academic Press, 1996.

[8] S.-K. Chang, Q. Y. Shi And C. W. Yan, Iconic Indexing by 2-D Strings, IEEE Trans. Pattern Anal Machine Intell, 9:3(1987), pp. 413-428.

[9] E. A. El-Kwae and M. R. Kabuka, A Robust Framework for Content-based Retrieval by Spatial Similarity in Image Databases, ACM Transactions on Information Systems, 17:2(1999), pp. $174-198$.

[10] M. Flickner, H. Sawhney and W. Niblack, et Al., Query by Image and Video Content: The QBIC System, IEEE Computer, 23-32, September 1995.

[11] V. N. GudivadA, On Spatial Similarity Measures for Multimedia Applications, Proceedings of SPIE - Storage and Retrieval for Still Images and Video Databases III, (SPIE, Bellingham, WA), San Jose, CA, 363-372, Feb. 9-10, 1995.

[12] V. N. Gudivada and V. V. Raghavan, Design and Evaluation of Algorithms for Image Retrieval by Spatial Similarity, ACM Transactions on Information Systems, 13:2(1995), pp. $115-144$.

[13] V. N. Gudivada and V. V. Raghavan, Content-based Image Retrieval Systems, IEEE Computer, 18-22, September 1995.

[14] T.-Y. Hou, P. Lui And M. Y. Chui, A Content-based Indexing Technique Using Relative Geometry Features, Proceedings of SPIE - Image Storage and Retrieval Systems, the International Society for Optical Engineering, Vol. 1662, 1992.

[15] A. Pentland, R. W. Picard and S. Sclroff, Photobook: Content-based Manipulation of Image Databases, Proceedings of SPIE - Storage and Retrieval for Image and Video Database II, (SPIE, Bellingham, WA), San Jose, CA, 34-47, Feb. 6-10, 1994.

[16] A. P. Sistla, C. Yu and R. Haddad, Reasoning about Spatial Relationships in Picture Retrieval Systems, Proceedings of the 20th International Conference on Very Large Databases, Santiago, Chile, 570-581, 1994.

[17] Q.-L. Zhang, A Unified Framework for Iconic Indexing of Spatial Relationships in Image Databases, Ph.D. Thesis, Department of Mathematics, Statistics, and Computer Science. University of Illinois. Chicago, 1996.

[18] Q.-L. Zhang, S.-K. Chang and S. S.-T. YAU, A Unified Approach to Indexing Images in Image Databases, Proceedings of the First International Workshop on Image Databases and Multi-Media Search, Amsterdam, The Netherlands, 99-106, August 22-23, 1996.

[19] Q.-L. Zhang, S.-K. Chang and S. S.-T. Yau, A Unified Approach to Iconic Indexing, Retrieval, and Maintenance of Spatial Relationships in Image Databases, Journal of Visual Communication and Image Representation, Special Issue on Indexing, Storage, Retrieval and Browsing of Images and Video, Academic Press, 7:4(1996), pp. 307-324.

[20] Q.-L. Zhang, S.-K. Chang and S. S.-T. YAu, An Experimental Result in Image Indexing using GEP-2D Strings, Image Databases and Multimedia Search (A. Smeulders and R. Jain, eds.), World Scientific Pub. Co., 127-146, 1997.

[21] Q.-L. Zhang, S.-K. Chang and S. S.-T. Yau, The Consistency Problem on Content-based Pictorial Description in Pictorial Database Systems, Communications in Information and Systems, International Press, 1:2(2001), pp. 225-240.

[22] Q.-L. Zhang and S. S.-T. Yau, A New Iconic Indexing for $2 D$ and $3 D$ Scenes, Proceedings of the 2nd Chinese World Congress on Intelligent Control and Intelligent Automation 
(CWCICIA 97), Xi'an, P. R. China, 1667-1672, June 23-27, 1997.

[23] Q.-L. Zhang And S. S.-T. Yau, Finding Minimal and Maximal Sets of Spatial Relationships in Pictorial Retrieval Systems, Submitted for Publication. 